\title{
Abdominal Pain, CTCAE
}

National Cancer Institute

\section{Source}

National Cancer Institute. Abdominal Pain, CT CAE. NCI Thesaurus. Code C143255.

A disorder characterized by a sensation of marked discomfort in the abdominal region. 